\title{
Suppression of Collapse for Spiraling Elliptic Solitons
}

\author{
Anton S. Desyatnikov, ${ }^{1}$ Daniel Buccoliero, ${ }^{1}$ Mark R. Dennis,${ }^{2}$ and Yuri S. Kivshar ${ }^{1}$ \\ ${ }^{1}$ Nonlinear Physics Center, Research School of Physics and Engineering, The Australian National University, \\ Canberra ACT 0200, Australia \\ ${ }^{2}$ H. H. Wills Physics Laboratory, University of Bristol, Tyndall Avenue, Bristol BS8 1TL, United Kingdom
}

(Received 24 August 2009; published 5 February 2010)

\begin{abstract}
We reveal that orbital angular momentum can suppress catastrophic self-focusing in nonlinear Kerr media supporting stable spiraling solitons with an elliptic cross section. We discuss the necessary requirements for observation of this effect with coherent optical and matter waves.
\end{abstract}

A balance between self-focusing and diffraction of light in two transverse dimensions is known to support the formation of optical spatial solitons [1], such as the bellshaped (fundamental) solitons [2], which undergo catastrophic collapse in bulk Kerr media [3-5]. Different suggestions have been made on how to arrest the collapse, e.g., by introducing alternating focusing-defocusing layers [6], nonlocal response [7], optical gain [8], or magneto-optic response [9]. The problem remains unsolved for pure Kerr media, however, although nonparaxial coupling of polarization components was shown to provide stabilization to circularly polarized beams [10].

The fundamental optical soliton can be stable in other Kerr-type media, e.g., in saturable photorefractive crystals [11], where inherent anisotropy can elliptically deform an otherwise radially symmetric transverse profile [12]. Elliptic self-trapping was also achieved by introducing anisotropy either into diffraction, using spatial anisotropy of the correlation function [13] of partially coherent elliptic solitons [14], or into nonlocal thermal response, controlled by rectangular boundaries, for coherent elliptic solitons [15]. In isotropic bulk nonlinear media, elliptically shaped coherent beams experience quasiperiodic oscillations around the radially symmetric soliton profile [16,17], and it is generally believed that elliptic solitons do not exist [14].

In this Letter we approach this long-standing problem from a completely different perspective and reveal that elliptic solitons can exist as spiraling beams being supported by the optical orbital angular momentum (OAM) [18]. Spiraling solitons [19] carrying OAM are usually associated with optical vortices $[20,21]$ and related ringshaped self-trapped beams [22]. At the same time, the vortex-free beams with nonzero OAM are well known in linear media, such as an elliptically shaped beam focused by a tilted cylindrical lens [23]. We demonstrate that the contribution of OAM to the dynamics of elliptic beams in nonlinear self-focusing media is twofold. First, it strengthens effectively the diffraction against self-focusing and can suppress collapse in Kerr media. Second, it preserves the elliptic profile of stably rotating solitons in optical media with collapse-free nonlinearities.
We start with a variational approach [24] of oscillating elliptic self-trapped beams [16] and consider the nonlinear Schrödinger equation with the Lagrangian $L=\frac{i}{2} \int\left\{E^{*} E_{z}-\right.$ $\left.E E_{z}^{*}\right\} d \mathbf{r}-H$, and Hamiltonian

$$
H=\int\left(|\nabla E|^{2}-\frac{1}{2}|E|^{4}\right) d \mathbf{r} .
$$

We introduce a trial function,

$$
E(x, y, z)=A(z) G[X / b(z)] G[Y / c(z)] \exp (i \Psi),
$$

with the Gaussian envelope $G(t)=\exp \left(-t^{2} / 2\right)$ and phase $\Psi=B(z) X^{2}+\Theta(z) X Y+C(z) Y^{2}+\varphi(z)$. The main novelty here with respect to previously considered nonrotating elliptic beams [16] is the asymmetric phase factor $\Theta(z)$, conjugated with the angle $\theta(z)$ of the rotating frame, $X=$ $x \cos \theta+y \sin \theta$ and $Y=-x \sin \theta+y \cos \theta$.

Below, we consider beams focused at $z=0$ by a cylindrical lens upon entry into the nonlinear media, and thus our boundary conditions for phase read $\left\{B_{0}, C_{0}, \varphi_{0}\right\}=0$, where subscript 0 indicates initial value, e.g., $B_{0}=B(0)$. The value $\Theta_{0} \sim(\kappa / 2 f) \sin 2 \alpha$ depends on the angle $\alpha$ between the major axis of the elliptically shaped beam with wave number $\kappa$ and the cylindrical lens of focal length $f$, and is independent of the other beam parameters [23].

Deriving variational equations, we obtain first integrals of motion: the power, $P=\pi A^{2} b c$, and the OAM, $M=$ $P \Theta\left(b^{2}-c^{2}\right) / 2$, as well as equations for the conjugated variables, $\quad \dot{b}=4 b B, \quad \dot{c}=4 c C, \quad$ and $\dot{\theta}=2 \Theta\left(b^{2}+\right.$ $\left.c^{2}\right) /\left(b^{2}-c^{2}\right)$. Dots indicate derivatives with respect to the evolution variable $z$, so $\{\dot{P}, \dot{M}, \dot{H}\}=0$. In the following it is convenient to introduce the spatial scale $a(z), a^{2}=$ $\left(b^{2}+c^{2}\right) / 2$, and the ellipticity parameter $\epsilon(z)=$ $\tan ^{-1}(c / b)$, then $b=\sqrt{2} a \cos \epsilon$ and $c=\sqrt{2} a \sin \epsilon$. The system can be cast in the Hamiltonian form,

$$
\begin{gathered}
H=\frac{P}{2}\left(\frac{\dot{a}^{2}}{2}+\frac{\mu}{a^{2}}\right), \quad \dot{\mu}=0, \\
\mu=\frac{a^{4} \dot{\epsilon}^{2}}{2}+\frac{4-p \sin 2 \epsilon}{2 \sin ^{2} 2 \epsilon}+\frac{2 \sigma^{2}}{\cos ^{2} 2 \epsilon},
\end{gathered}
$$

with $\quad p \equiv P / \pi=A^{2} a^{2} \sin 2 \epsilon$ and $\sigma \equiv M / P=$ $a^{2} \Theta \cos 2 \epsilon$. For a beam with waist at $z=0,\left\{\dot{a}_{0}, \dot{\epsilon}_{0}\right\}=0$, 
from Eq. (3) we obtain $a^{2}(z)=a_{0}^{2}\left(1+z^{2} / z_{0}^{2}\right)$, here $z_{0}=$ $a_{0}^{2} / \sqrt{2 \mu}$. Note the non-negative contribution of OAM to diffraction, $\sim \sigma^{2}$, counteracting self-focusing, $\sim-p$.

Collapse of circular beams in Kerr media.-Before analyzing spiraling solitons, we recall the details of the collapse of circular beams with $b=c=a(\epsilon \equiv \pi / 4)$ and zero OAM, $\sigma=0$. The variational Eqs. (3) and (4) predict that the integral of motion $\mu$ is a critical parameter of selffocusing, namely, for $\mu>0$ the beam diffracts, for $\mu<0$ the beam collapses at the distance $\left|z_{0}\right|$, and for $\mu=0$ the stationary (soliton) solution exists with $P=4 \pi \simeq 12.6$; cf. the critical power of self-focusing $P_{c}=11.7$ [25].

Variational solutions are obtained by choosing an arbitrary spatial scale $a_{0}$ because in Kerr media Eq. (1) the general solution is defined up to the arbitrary scale factor; i.e., if $E(x, y, z)$ is a solution then $a_{0}^{-1} E\left(x / a_{0}, y / a_{0}, z / a_{0}^{2}\right)$ is another solution. It follows that for the Gaussian ansatz Eq. (2) the only parameter which defines the evolution of circular beams is the power $P$. As an example we consider supercritical power $p=28$ and choose $a_{0}=5$ for convenience of numerical simulations of Eq. (1). Corresponding parameter $\mu=-12$ and variational approach predicts that the beam will collapse at the distance $\left|z_{0}\right|=5.1$.

Although predicting the event of collapse, the variational solution does not describe actual dynamics adequately, as seen from comparison in Fig. 1. The reason is that, as shown in Fig. 1(c), the collapsing beam develops a transverse profile very different from a Gaussian, namely, a sharp-peaked collapsing Townes soliton on a wide strongly diffracting background [4]. As a result, the actual Townes spike collapses at the distance $z \gtrless 2.3$. Nevertheless, the variationally obtained parameters have the direct meaning of averaged parameters of the actual profile. To demonstrate this numerically we monitor the mean radius of the beam, $a^{2}=\int\left(x^{2}+y^{2}\right)|E|^{2} d \mathbf{r} / P$, and its amplitude, $A^{2}=$ $p / a^{2}$, the results shown by dots in Fig. 1(a) and 1(b) and they follow the variational predictions (thin lines) with remarkable accuracy.

Spiraling elliptic beams in Kerr media.-In addition to a circular soliton with $\sigma=0$, the variational Eqs. (3) and (4) predict the existence of a novel kind of solution, a spiraling elliptic soliton with $\sigma \neq 0$, obtained for $\{\dot{a}, \dot{\epsilon}, H, \mu, \partial \mu / \partial \epsilon\}=0$. Because the predicted envelope (a) Amplitude

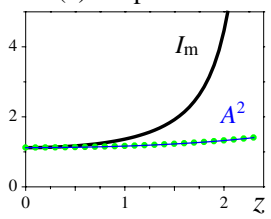

(b) Radius

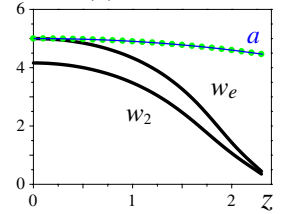

(c) Intensity

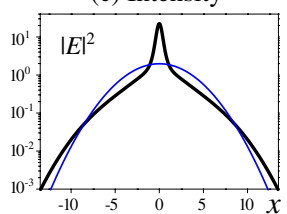

FIG. 1 (color online). Collapse of a circular beam with power $P=28 \pi=7.52 P_{c}$; the variational solution (thin lines) is compared to numerical data (thick lines and dots). Collapse is evident in (a) from the diverging peak intensity, $I_{m}=$ $\max |E|^{2}$, and in (b) from collapsing radii, $w_{2}\left(\right.$ at $I_{m} / 2$ ) and $w_{e}$ (at $I_{m} / e$ ). (c) Spatial profile of intensity at $z=2.3$. is stationary in the rotating frame, the rotation velocity is constant, $\omega \equiv \dot{\theta}_{s}=2 \sigma_{s} / a_{0}^{2} \cos ^{2} 2 \epsilon_{s}$, here the subscript $s$ denotes soliton solutions. Solitons are conveniently represented by parametrizing with $\epsilon_{s}$, namely, the soliton power, $p_{s}=16 / \sin 2 \epsilon_{s}\left(3-\cos 4 \epsilon_{s}\right)$, the OAM, $\sigma_{s}^{2}=$ $2 \cos ^{4} 2 \epsilon_{s} / \sin ^{2} 2 \epsilon_{s}\left(3-\cos 4 \epsilon_{s}\right)$, and the propagation constant, $k_{s} \equiv \dot{\varphi}_{s}=\left(5+\cos 4 \epsilon_{s}\right) / a_{0}^{2} \sin ^{2} 2 \epsilon_{s}\left(3-\cos 4 \epsilon_{s}\right)$.

The following analysis exploits the fact that the OAM is an additional, physically independent parameter of the elliptic soliton, defined by the tilt $\alpha$ of a given cylindrical lens; i.e., the parameters $\Theta_{0}$ and $\sigma$ can be set in an experiment independent of the beam envelope $p$ and $\boldsymbol{\epsilon}_{0}$. Therefore, for a given soliton solution $\left\{\epsilon_{s}, p_{s}, \sigma_{s}\right\}$, we can introduce an additional tilt factor $\eta$ and compare beams with the same power $p_{s}$ and envelope $\epsilon_{s}$ but different values of OAM, $\Theta_{0}=\eta \Theta_{s}$ and $\sigma=\eta \sigma_{s}$. In particular, the variational soliton solution is recovered for $\eta=1$, and for $\eta=0$ we observe the collapse of elliptic beams with zero OAM studied earlier [26]. An example for $p_{s}=20$ $\left(P=5.37 P_{c}, \epsilon_{s}=0.18, \sigma_{s}=2.32, \Theta_{s}=0.1\right)$, shown in Figs. 2-4, uncovers a physical effect of the nonzero OAM and spiraling, $\eta>0$, on the dynamics of collapse.

Increasing the OAM of an elliptic beam with a given initial envelope slows down the collapse, as seen from the dynamics of the peak intensity $I_{m}$ in Fig. 2(a) and two halfwidths in Fig. 2(b), defined at the level $I_{m} / e$ in the horizontal, $w_{x}$, and vertical, $w_{y}$, directions. When the twist of an elliptic beam $\Theta_{0}$, and the OAM $\sigma$, exceeds some threshold value $\eta>\eta_{c}$, the collapse is completely eliminated and the beam diffracts slowly. For our representative example in Figs. 2(a) and 2(b) the separatrix value is found to be $3<\eta_{c}<3.5$, in contrast to variational solutions predicting $\eta_{c}=1$. We performed simulations similar to Figs. 2(a) and 2(b) but with different values of beam power and we approximate the soliton existence curve as a boundary separating domains of diffraction and collapse; see the solid line in Fig. 2(c). The actual value of OAM required to suppress collapse is found to be much larger than that predicted by variational approach (dashed line). Nevertheless, as pointed out in Ref. [23], the very high OAM can be achieved in experiment, $\sigma \sim 10^{4}$, although the relatively low values of $\sigma=6-12$ are required to eliminate the collapse of the beams with supercritical powers $P=(5-8) P_{c}$;
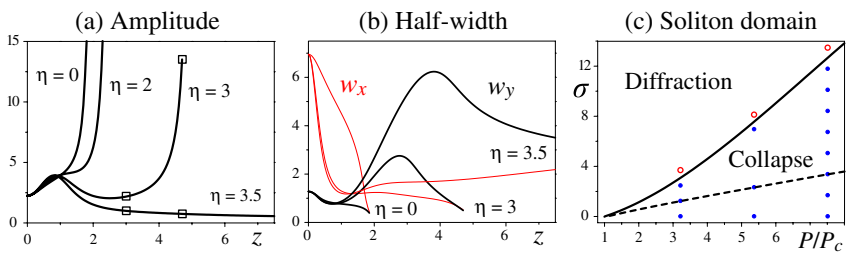

FIG. 2 (color online). (a),(b) Dynamics of spiraling elliptic beams in Kerr media, $P=20 \pi=5.37 P_{c}$. (c) Optical angular momentum versus power showing the domains of diffraction and collapse. Solid line: Approximate boundary between numerical solutions with and without collapse (dots and circles). Dashed line: Variational solution for the soliton family, $\sigma_{s}$. 

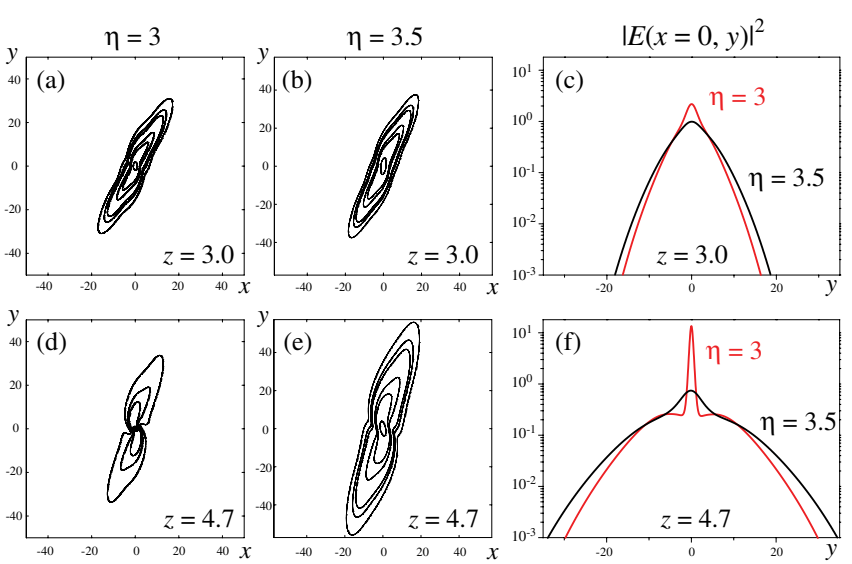

FIG. 3 (color online). Intensity profiles of elliptic beams as indicated by squares in Fig. 2(a). There are six contour lines in (a),(b),(d),(e) at the levels $(0.001,0.005,0.01,0.05,0.1,0.5)$ $I_{m}$.

see Fig. 2(c). As a realistic example we consider experiments in BK7 glass [4] with the beam of power $p=3.5$, wavelength $\lambda=800 \mathrm{~nm}$, transverse scale $a_{0} \simeq 0.5 \mathrm{~mm}$, and ellipticity $\epsilon_{0} \simeq 1.25$. In accordance with Fig. 2(c) the collapse will be suppressed for $\sigma=4$, which requires a cylindrical lens with the focal length $f \simeq 20 \mathrm{~cm}$ tilted by $\alpha=45^{\circ}$ with respect to elliptic beam profile.

A more detailed analysis of the process of collapse suppression is presented in Fig. 3, where we compare two beams from different domains $\eta \gtrless \eta_{c}$ close to the separatrix. To stress their differences, we plot the low-level contours in Figs. 3(a), 3(b), 3(d), and 3(e) and use logarithmic scale in Figs. 3(c) and 3(f). As seen in Fig. 3(c) at $z=3$, before the collapse of supercritical beam, both beams have similar envelopes. We use the term "supercritical" here for the beam with power exceeding the diffraction-collapse threshold for a given value of OAM, $\sigma=\eta \sigma_{s}$. With further propagation at $z=4.7$, both beams rotate in the anticlockwise direction because the OAM is positive, $\sigma>0$. The supercritical beam with $\eta=3$ develops characteristic spike of circularly symmetric Townes profile [4], see Fig. 3(d) and 3(f), while the subcritical beam with $\eta=3.5$ in 3(e) and 3(f) only diffracts without strong reshaping. Remarkably, as shown in Fig. 3(f), the background of the supercritical beam still diffracts very fast, despite the larger OAM of the subcritical beam. We conclude that the subcritical spiraling improves the balance between diffraction and self-focusing which leads to the trapping of the beam as a whole, in contrast to the supercritical beam with the central part collapsing and the background strongly diffracting.

Further dynamics of the diffracting beam with $\eta>\eta_{c}$ is shown in Fig. 4. The slow rotation and spreading of the beam is evident, and we also notice almost self-similar profiles with characteristic "propellerlike" transverse shape in their tails as a result of spatial twist. This observation strongly suggests that the beam is close to the soliton solution. However, as with the circular beams in
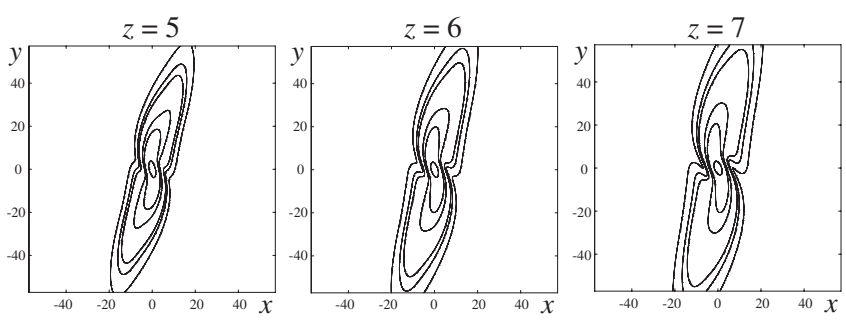

FIG. 4. Further propagation of subcritical beam with $\eta=3.5$ in Figs. 3(b) and 3(e).

Kerr media, such solitons require exact balance between self-focusing and diffraction which is unstable to small perturbations.

Spiraling elliptic solitons in saturable media.-In collapse-free Kerr-type media stable solitons survive small perturbations by compensating them with internal oscillations $[16,17]$. Here we choose saturable medium as a representative example to establish existence and stability of spiraling elliptic solitons. Rather than by Eq. (1), the Hamiltonian of this model is described by $H=\int\left\{|\nabla E|^{2}-\right.$ $\left.|E|^{2}+\log \left(1+|E|^{2}\right)\right\} d \mathbf{r}$. Using the variational approach with the ansatz Eq. (2), we derive the following effective-particle representation:

$$
\begin{gathered}
4\left(\frac{H}{P}+1\right)=\frac{1}{2} \dot{b}^{2}+\frac{1}{2} \dot{c}^{2}+\Pi(b, c), \\
\Pi(b, c)=\frac{2}{b^{2}}+\frac{2}{c^{2}}+\frac{8 \sigma^{2}\left(b^{2}+c^{2}\right)}{\left(b^{2}-c^{2}\right)^{2}}-\frac{4 b c}{p} \operatorname{Li}_{2}\left(-\frac{p}{b c}\right),
\end{gathered}
$$

with two independent parameters, $\sigma$ and $p$.

An important difference from the Kerr model is the absence of the arbitrary scaling $a_{0}$; i.e., the spiraling solitons belong to a two-parameter family [27]. In addition to the usual soliton constant $k$ (or the power $p$ ), the spiraling solitons are parametrized by the angular velocity $\omega$ (or the OAM $\sigma$ ). Solitons can be found as the minima of the potential $\Pi(b, c)$; one example is shown in Fig. 5 for $\sigma=$ 0.35 and $p=127.32$ (other parameters are $b_{s}=4.26$, $c_{s}=2.13, \Theta_{s}=0.052$, and $\left.\omega_{s}=0.17\right)$. The isosurface of intensity of spiraling soliton shows homogeneous rotation free from oscillations. We also compare two halfwidths, $w_{x}$ and $w_{y}$, with corresponding variational solution, $w_{x} \rightarrow\left(b_{s}^{-2} \cos ^{2} \omega_{s} z+c_{s}^{-2} \sin ^{2} \omega_{s} z\right)^{-1 / 2}$ and $w_{y} \rightarrow$ $w_{x}\left(b_{s} \leftrightarrow c_{s}\right)$, and find an excellent agreement.

We should mention that closer to the Kerr limit, i.e., for low powers, we observe the development of low-intensity oscillating tails which indicate the appearance of dispersive waves radiated by the soliton (not shown). This picture is consistent with the linear stability analysis [17], namely, the absence of oscillatory modes of the fundamental solitons with $k_{s} \lesssim 0.5$. The radiative tails take a negligible portion of power from the soliton while the amount of radiated OAM can be more significant. The reduction of OAM in the main soliton leads to the slow reduction of ellipticity of the transverse rotating profile. Nevertheless, 

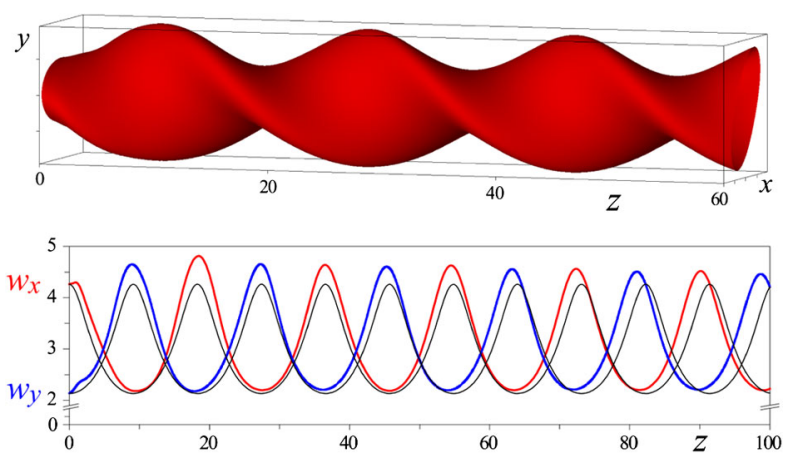

FIG. 5 (color online). Isointensity plot at the level $I_{m} / 2$ of elliptic soliton with $k_{s}=0.7$ and $I_{m}=14$ in saturable media. Numerically obtained half-widths $w_{x}$ and $w_{y}$ (thick lines) are compared to variational solution (thin lines).

this process is practically eliminated in the regime of noticeable saturation, as in the example in Fig. 5.

In experiment, the soliton spiraling is observed in the samples of limited length [27], because the conservation of OAM requires isotropic media, and it is violated by anisotropy of photorefractive crystals or absorption in vapors of two-level atoms. The stable elliptic solitons can be generated, for typical conditions of fundamental solitons, as an output elliptic beam rotating as a function of the tilt $\alpha$ of the input cylindrical lens, which changes the OAM $\sigma$ and the rate of spiraling $\omega$ from zero (for $\alpha=0$ ) to a maximal value (for $\alpha=\pi / 4$ ).

Similar to optics, collapse of matter waves can be controlled by changing nonlinearity: manipulating the contact atom-atom interactions via a Feshbach resonance [28] or long-range dipole-dipole interactions via the trap geometry [29]. At the same time, the asymmetric traps were employed to rotate repulsive condensates and achieve vortex generation [30]. Our results suggest to combine these two well-established techniques and explore, theoretically and experimentally, the possibility to suppress the collapse of the attractive Bose-Einstein condensates in rotating traps.

In conclusion, we have revealed that spiraling of selftrapped beams is an efficient mechanism for suppressing catastrophic collapse in Kerr media and supporting stable elliptic solitons. Our concept can be extended to other nonlinearities such as nonlocal and quadratic. For spatiotemporal problems, our approach allows us to introduce the "twisted pulses" [31] as stable light bullets in collapsefree media [32]. In Kerr media, however, light bullets are always unstable [25], because the contribution to the Hamiltonian from the self-focusing term, $\sim-p a^{-2} \tau^{-1}$, dominates both the diffraction, $\sim a^{-2}$, and the dispersion, $\sim \tau^{-2}$; here, $\tau$ is the duration of the pulse with energy $p$. The transverse spatial rotation with OAM $\sigma$ contributes the term similar to diffraction, $\sim \sigma^{2} a^{-2}$, and thus it cannot counterbalance the simultaneous spatiotemporal collapse. Nevertheless, the effect of the OAM on the collapse dynamics is expected to be rather significant.
[1] Yu. S. Kivshar and G.P. Agrawal, Optical Solitons: From Fibers to Photonic Crystals (Academic, New York, 2003).

[2] R. Y. Chiao, E. Garmire, and C.H. Townes, Phys. Rev. Lett. 13, 479 (1964).

[3] L. Berge, Phys. Rep. 303, 259 (1998).

[4] K. D. Moll, A. L. Gaeta, and G. Fibich, Phys. Rev. Lett. 90, 203902 (2003).

[5] Self-Focusing: Past and Present, edited by R. W. Boyd, S. G. Lukishova, and Y.R. Shen (Springer, New York, 2009).

[6] L. Berge et al., Opt. Lett. 25, 1037 (2000); M. Centurion et al., Phys. Rev. Lett. 97, 033903 (2006).

[7] O. Bang et al., Phys. Rev. E 66, 046619 (2002).

[8] S. Chen and J. M. Dudley, Phys. Rev. Lett. 102, 233903 (2009).

[9] Y. Linzon et al., Phys. Rev. Lett. 103, 053902 (2009).

[10] G. Fibich and B. Ilan, Phys. Rev. E 67, 036622 (2003).

[11] M. Segev et al., Phys. Rev. Lett. 68, 923 (1992).

[12] A. A. Zozulya et al., Europhys. Lett. 36, 419 (1996); P. Zhang et al., Opt. Express 15, 536 (2007).

[13] D. N. Christodoulides et al., Phys. Rev. Lett. 80, 2310 (1998); H. Buljan et al., Phys. Rev. E 68, 036607 (2003).

[14] E. D. Eugenieva, D. N. Christodoulides, and M. Segev, Opt. Lett. 25, 972 (2000); O. Katz et al., ibid. 29, 1248 (2004).

[15] C. Rotschild et al., Phys. Rev. Lett. 95, 213904 (2005).

[16] B. Crosignani and P. Di Porto, Opt. Lett. 18, 1394 (1993); A. W. Snyder and J. D. Mitchell, ibid. 22, 16 (1997); V. Tikhonenko, ibid. 23, 594 (1998).

[17] J. Yang, Phys. Rev. E 66, 026601 (2002).

[18] Optical Angular Momentum, edited by L. Allen, S. M. Barnett, and M.J. Padgett (Institute of Physics, Bristol, 2003).

[19] A. S. Desyatnikov, Yu. S. Kivshar, and L. Torner, Prog. Opt. 47, 291 (2005).

[20] J.F. Nye and M. V. Berry, Proc. R. Soc. A 336, 165 (1974); M. S. Soskin and M. V. Vasnetsov, Prog. Opt. 42, 219 (2001); M. R. Dennis, K. O'Holleran, and M. J. Padgett, ibid. 53, 293 (2009).

[21] V. I. Kruglov, Yu. A. Logvin, and V. M. Volkov, J. Mod. Opt. 39, 2277 (1992); W. J. Firth and D. V. Skryabin, Phys. Rev. Lett. 79, 2450 (1997).

[22] M. Soljacic and M. Segev, Phys. Rev. Lett. 86, 420 (2001); A. S. Desyatnikov and Yu. S. Kivshar, ibid. 88, 053901 (2002); A. S. Desyatnikov, A. A. Sukhorukov, and Yu. S. Kivshar, ibid. 95, 203904 (2005).

[23] J. Courtial et al., Opt. Commun. 144, 210 (1997).

[24] B. A. Malomed, Prog. Opt. 43, 71 (2002).

[25] Y. Silberberg, Opt. Lett. 15, 1282 (1990).

[26] V. P. Kandidov and V. Yu. Fedorov, Quantum Electron. 34, 1163 (2004).

[27] V. Tikhonenko, J. Christou, and B. Luther-Davies, J. Opt. Soc. Am. B 12, 2046 (1995); M. F. Shih, M. Segev, and G. Salamo, Phys. Rev. Lett. 78, 2551 (1997).

[28] E. A. Donley et al., Nature (London) 412, 295 (2001).

[29] T. Koch et al., Nature Phys. 4, 218 (2008).

[30] K. W. Madison et al., Phys. Rev. Lett. 84, 806 (2000); J. R. Abo-Shaeer et al., Science 292, 476 (2001).

[31] A. Desyatnikov, A. Maimistov, and B. Malomed, Phys. Rev. E 61, 3107 (2000).

[32] B. A. Malomed et al., J. Opt. B 7, R53 (2005). 